Supporting Information

\title{
Effect of Composition on the Spin Relaxation of Lead Halide Perovskites
}

Meng Zhou, Julio S. Sarmiento, Chengbin Fei, Xinwen Zhang, He Wang*

Department of Physics, University of Miami, Coral Gables, Florida 33146, USA

*To whom correspondence should be addressed. Email: hewang@miami.edu

Materials and Methods.

S1. Scanning electron microscope (SEM) image of perovskite thin films.

S2. Determination of exciton binding energy of different perovskite thin films.

S3. Spin dynamics of perovskite thin films probed at ESA band.

S4. Fitting of electron and hole spin lifetimes.

S5. Carrier lifetime measurements.

S6. UV-vis absorption spectra and spin dynamics of $\mathrm{MAPbIBr} 2$ and $\mathrm{MAPbI}_{2} \mathrm{Br}$.

S7. Spin dynamics of $\mathrm{MAPbI}_{3}$ thin film at different temperatures.

S8. Spin dynamics of $\mathrm{CsPbI}_{3}$ thin film at different temperatures.

S9. Spin dynamics of MAPbBr3 thin film at different temperatures.

S10. Spin dynamics of $\mathrm{CsPbBr}_{3}$ thin film at different temperatures.

S11. Repeated low temperature measurement on MAPbI3.

S12. Low Temperature Photoluminescence of $\mathrm{MAPbI}_{3}$ and MAPbBr 3 thin films. 


\section{Materials and Methods}

\section{Preparation of MAPbI, MAPbI ${ }_{2} \mathrm{Br}_{3}$ MAPbIBr2 and MAPbBr3 thin films.}

$31.8 \mathrm{mg}$ (0.2 mmol) MAI, $92.2 \mathrm{mg}(0.2 \mathrm{mmol}) \mathrm{PbI}_{2}$ and $14 \mu \mathrm{L}$ DMSO were dissolved in anhydrous DMF $(186 \mu \mathrm{L})$ for MAPbI 3 .

$22.4 \mathrm{mg}(0.2 \mathrm{mmol}) \mathrm{MABr}$, $92.2 \mathrm{mg}(0.2 \mathrm{~m} \mathrm{~mol}) \mathrm{PbI}_{2}$ and $14 \mu \mathrm{L}$ DMSO were dissolved in anhydrous DMF $(186 \mu \mathrm{L})$ for $\mathbf{M A P b I}_{2} \mathbf{B r}$.

$31.8 \mathrm{mg}$ (0.2 mmol) MAI, $74.3 \mathrm{mg}(0.2 \mathrm{mmol}) \mathrm{PbBr}_{2}$ and $14 \mu \mathrm{L}$ DMSO were dissolved in anhydrous DMF $(186 \mu \mathrm{L})$ for MAPbIBr2.

$22.4 \mathrm{mg}$ (0.2 mmol) $\mathrm{MABr}, 73.4 \mathrm{mg}(0.2 \mathrm{mmol}) \mathrm{PbBr}_{2}$ and $14 \mu \mathrm{L}$ DMSO were dissolved in anhydrous DMF $(186 \mu \mathrm{L})$ for MAPbBr3.

$50 \mu \mathrm{L}$ of well-dissolved solution (aging for 6 hours) was dropped onto the pre-cleaned glass substrate for spin coating at $4000 \mathrm{rpm}$ for $30 \mathrm{~s}$. $50 \mu \mathrm{L}$ ethyl acetate was dropped as antisolvent onto the substrate after spinning 5-7 s. These films were finally thermally annealed at $100^{\circ} \mathrm{C}$ for $30 \mathrm{~min}$. Finally, a thin layer $(\sim 50 \mathrm{~nm})$ of PMMA was fabricated on the above perovskite films.

Preparation of $\mathrm{CsPbI}_{3}$ thin films. The $\mathrm{CsPbI}_{3}$ precursor was prepared by dissolving $126.8 \mathrm{mg}$ $\left(\mathrm{CH}_{3}\right)_{2} \mathrm{NHPbI}_{\mathrm{x}}$ powder and $41.6 \mathrm{mg} \mathrm{CsI}$ in $0.2 \mathrm{~mL} \mathrm{DMF}$ at room temperature and aged overnight. The $\left(\mathrm{CH}_{3}\right)_{2} \mathrm{NHPbI}_{\mathrm{x}}$ powder (previously misunderstood as $\mathrm{HPbI}_{3}$ ) was synthesized according to the literature. ${ }^{1} 50 \mu \mathrm{L}$ precursor solution was spin coated onto cleaned substrate with a speed of $2500 \mathrm{rpm}$ for $30 \mathrm{~s}$. Then, the films were annealed at $185^{\circ} \mathrm{C}$ for $12 \mathrm{~min}$, followed by fast cooling at room temperature. Finally, a thin layer $(\sim 50 \mathrm{~nm})$ of PMMA was fabricated on the above perovskite films.

Preparation of $\mathrm{CsPbBr}_{3}$ thin films. The perovskite solution was prepared by dissolving $\mathrm{PbBr}_{2}$ (22.02mg, $0.3 \mathrm{mmol})$ and $\mathrm{CsBr}(12.77 \mathrm{mg}, 0.3 \mathrm{mmol})$ in DMSO $(200 \mu \mathrm{L})$, followed by aging for 3 hours. Then the solution $(50 \mu \mathrm{L})$ was spin coated on the substrate at $3000 \mathrm{rpm}$ for $30 \mathrm{~s}$ and annealed at $70{ }^{\circ} \mathrm{C}$ for $10 \mathrm{~min}$. Finally, a thin layer $(\sim 50 \mathrm{~nm})$ of PMMA was fabricated on the above perovskite films.

All of the film preparation was carried in a nitrogen-filled glove box. 


\section{Circular polarized transient absorption spectroscopy}

The pump beam was generated in a collinear optical parametric amplifier (Light conversion) pumped by the $800 \mathrm{~nm}$ output of an amplified Ti:sapphire laser (Coherent Astrella, $5 \mathrm{kHz}$ ) and chopped at $2.5 \mathrm{kHz}$. The circular polarized transient absorption measurements were performed on a commercial spectrometer (Helios Fire, Ultrafast System). A small portion of the laser fundamental was focused onto a sapphire plate to produce supercontinuum in the visible region, which overlapped in time and space with the pump. A $\lambda / 2$ waveplate, a Glan Tylor prism and a $\lambda / 4$ waveplate were used to change the pump and probe pulses from linear polarization to left and right circular polarizations. Multiple wavelength spectra were collected by a spectrometer with CMOS linear array detector. The pump fluence was kept as low as $2 \mu \mathrm{J} / \mathrm{cm}^{2}$ and the carrier densities are in the order of $10^{-17} \mathrm{~cm}^{-3}$ so that there was no bimolecular recombination. The intensity of white continuum (400-800 nm) was attenuated to less than $15 \mu \mathrm{W}$. All measurements were taken in the vacuum chamber of a cryostat (Janis Research Company). For $\mathrm{MAPbI}_{3}$ and $\mathrm{CsPbI}_{3}$, we pumped at $710 \mathrm{~nm}$. For $\mathrm{MAPbBr}_{3}$ and $\mathrm{CsPbBr}_{3}$, we pumped at $490 \mathrm{~nm}$. The pump energy is slightly above the bandgaps of perovskite films so that the effect of hot carrier is minimized.

\section{Sample characterization}

The UV-vis absorption spectra were measured using an Agilent Cary $60 \mathrm{UV}-\mathrm{vis}$ spectrometer. Scanning electron microscope image was taken using a JSM-7100F Scanning Electron Microscope.

\section{Spin Relaxation for graphene}

In graphene, the Rashba splitting dominates and the spin lifetimes $\left(\tau_{\mathrm{s}}\right)$ for Elliot-Yafet (E-Y) and D'yakonov-Perel (D-P) can be written as: ${ }^{2} \tau_{s}^{E Y} \approx \frac{\left(v_{F} k_{F}\right)^{2}}{\Delta^{2}} \tau_{p}$ and $\tau_{s}^{D P} \approx \frac{1}{\Delta^{2} \tau_{p}}$, respectively. Here, $k_{F}$ and $v_{\mathrm{F}}$ are the Fermi velocity and momentum, respectively, $\Delta$ is the spin-orbital splitting energy, and $\tau_{\mathrm{p}}$ is the momentum relaxation time. One can find that in both Rashba and Dresselhaus effects, $\tau_{s}^{E Y} \propto \tau_{p}$ and $\tau_{s}^{D P} \propto \frac{1}{\tau_{p}}$. 


\section{S1. Scanning electron microscope (SEM) image of perovskite thin films.}

One can find that all of these four perovskite thin films show large grain size $(>100 \mathrm{~nm})$ and there are no significant pin holes in these films (Figure S1). As shown in Figure S1d, the quality of $\mathrm{CsPbBr}_{3}$ thin film is not as good as other three films. Previous work reported that the morphology of $\mathrm{CsPbBr}_{3}$ can be improved by adding polymers. ${ }^{3,4}$ However, to prevent the potential effect on the physical properties of $\mathrm{CsPbBr}_{3}$, we didn't use polymer in our film preparation.

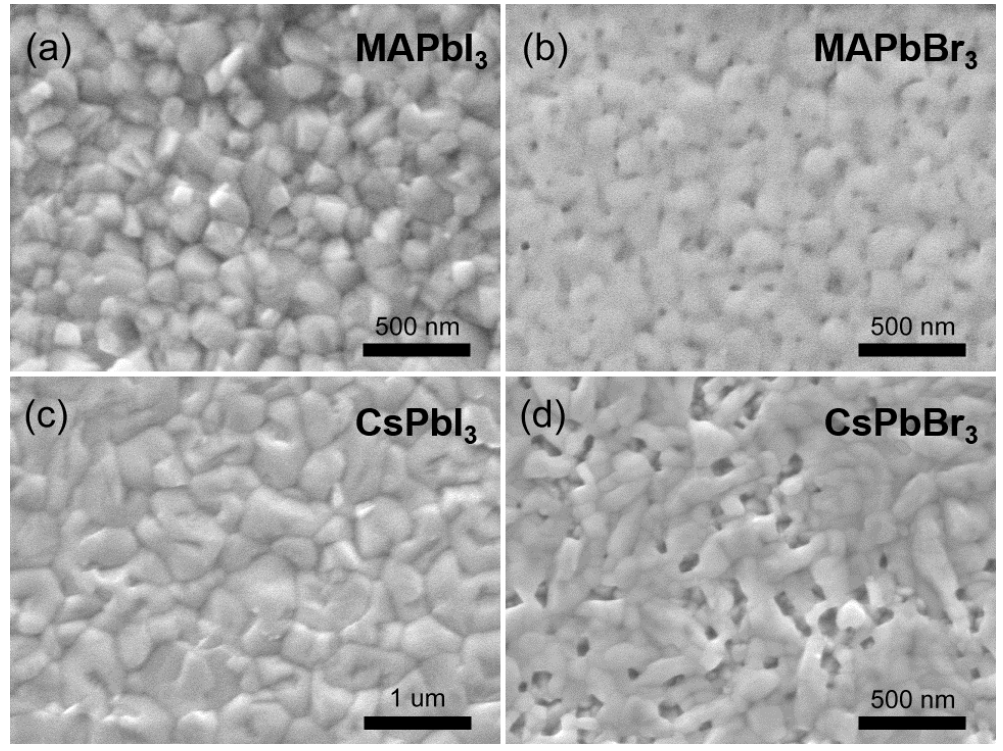

Figure S1. Scanning electron microscopy image of (A) $\mathrm{MAPbI}_{3}$; (B) $\mathrm{MAPbBr}_{3}$; (C) $\mathrm{CsPbI}_{3}$ and (D) $\mathrm{CsPbBr}_{3}$. 


\section{S2. Determination of exciton binding energy of different perovskite thin films.}

The absorption spectrum at the band edge is described by the following Elliot equation: ${ }^{5-7}$

$$
\alpha(\hbar \omega)=P_{c v}\left[\theta\left(\hbar \omega-E_{g}\right) \cdot\left(\frac{\pi e^{\pi x}}{\sinh (\pi x)}\right)\right]+R_{e x} \sum_{n=1}^{\infty} \frac{4 \pi}{n^{3}} \delta\left(\hbar \omega-E_{g}+\frac{R_{e x}}{n^{2}}\right)
$$

where $P_{c v}$ is a constant related to interband transition, $\theta\left(\hbar \omega-E_{g}\right)$ is the heaviside step function, $x$ is $\sqrt{R_{e x} /\left(\hbar \omega-E_{g}\right)}, \delta$ is a delta function. The first term refers to the continuum states and the second term represents the excitonic resonance. The first term in eq (1) can be simplified to:

$$
\alpha\left(\hbar \omega=E_{g}\right)=\alpha_{f} \cdot \frac{2 \pi R_{e x}^{1 / 2}}{\sqrt{\hbar \omega-E_{g}}}
$$

where $\alpha_{f}$ represents the absorption coefficient without coulomb interaction. The absorption spectra of four perovskite films can be fitted well using the equation (1) via homely written Matlab code and the results are shown in Figure S2.
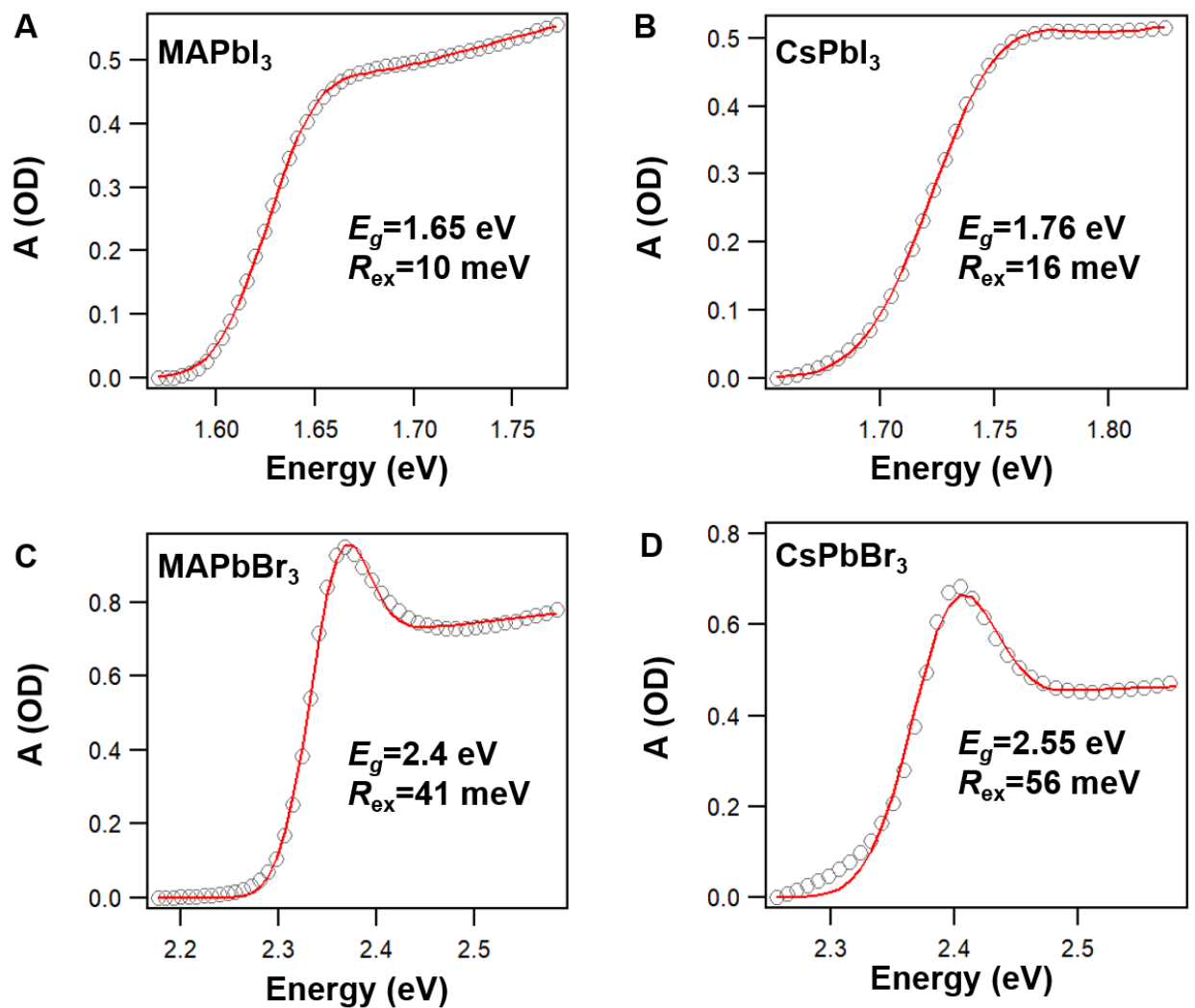

Figure S2. UV-vis absorption spectra and fit of (A) $\mathrm{MAPbI}_{3},(\mathrm{~B}) \mathrm{CsPbI}_{3},(\mathrm{C}) \mathrm{MAPbBr}_{3}$ and (D) $\mathrm{CsPbBr}_{3} . E_{g}$ represents bandgap and $R_{e x}$ represents exciton binding energy. 
S3. Spin dynamics of perovskite thin films probed at ESA band.
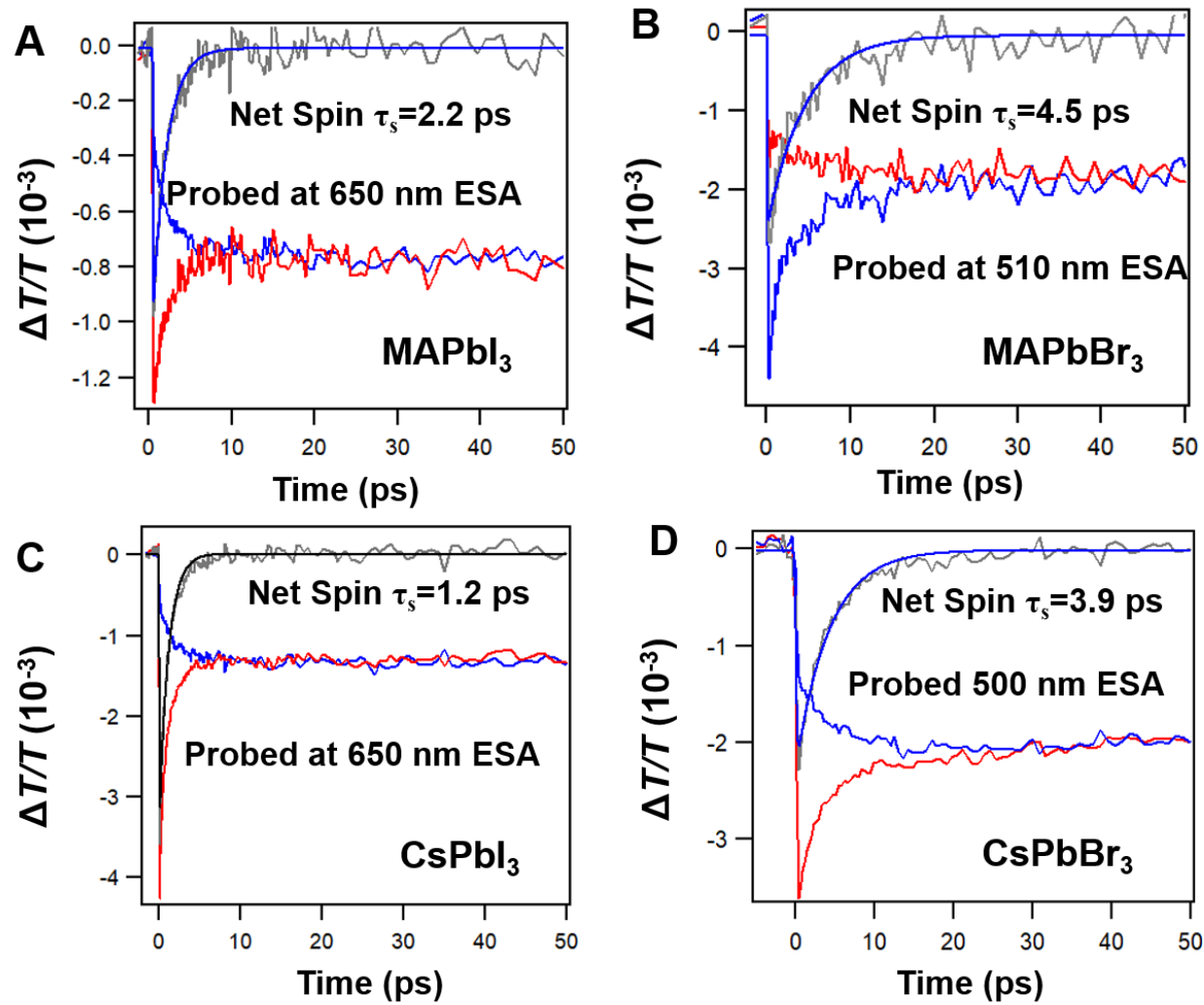

Figure S3. The co- and counter-circular polarized pump-probe kinetics with their difference of (A) $\mathrm{MAPbI}_{3}$, (B) $\mathrm{MAPbBr}_{3}$, (C) $\mathrm{CsPbI}_{3}$ and (D) $\mathrm{CsPbBr}_{3}$ at room temperature. The probe wavelength is chosen at the ESA band. 


\section{S4. Fitting of electron and hole spin lifetimes.}

To derive values for the electron spin lifetime and hole spin lifetime, we globally fitted the co-circular and counter-circular polarization pump-probe signals using equation (3) as our model. ${ }^{8}$ We fitted the data using the software MATLAB. Equation (3) describes the individual contributions of the electron and hole to the co-circular/counter-circular signal with $+/$ - denoting co-circular/counter-circular.

$$
\left(\frac{\Delta T}{T}\right)_{ \pm 1 / 2} \propto e^{-t / \tau_{c}}\left\{\left[1+\operatorname{erf}\left(\frac{t}{\tau_{0}}-\frac{\tau_{0}}{2 \tau_{c}}\right)\right]+\frac{1}{2} \sum_{i=e, h} e^{\tau_{0}{ }^{2} / \tau_{i}^{2}}\left(1+\operatorname{erf}\left(\frac{t}{\tau_{0}}-\frac{\tau_{0}}{\tau_{i}}\right)\right) e^{2 t / \tau_{i}}\right\}
$$

where $\tau_{\mathrm{c}}$ is the spin-independent carrier relaxation time, $\tau_{0}$ is laser temporal pulse width parameter (Gaussian pulse), $\tau_{\mathrm{e}}$ and $\tau_{\mathrm{h}}$ represent electron and hole spin lifetime, respectively.
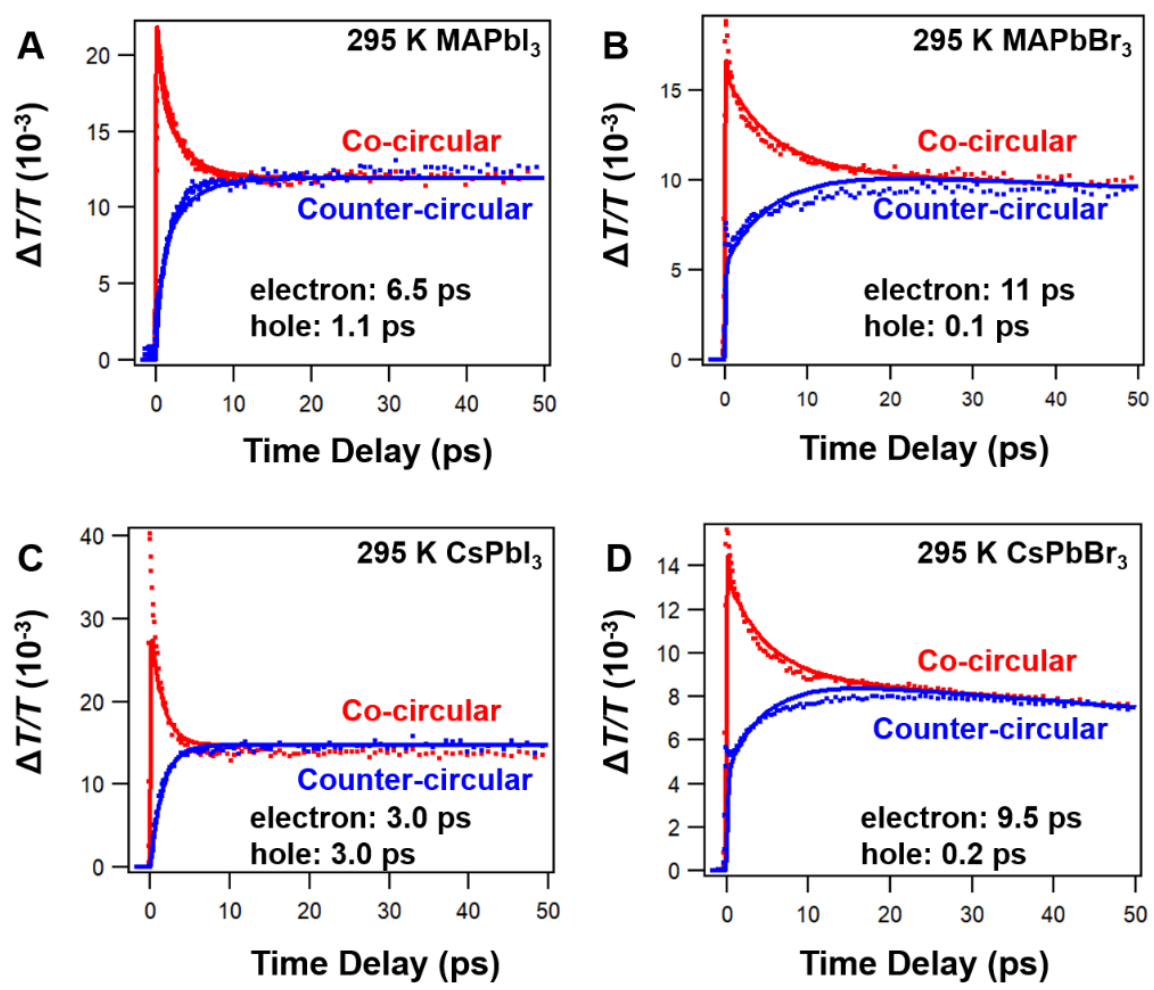

Figure S4. The co- and counter-circular polarized pump-probe kinetics with fitting on the electron and hole spin lifetime of (A) $\mathrm{MAPbI}_{3}$, (B) $\mathrm{MAPbBr}_{3}$, (C) $\mathrm{CsPbI}_{3}$ and (D) $\mathrm{CsPbBr}_{3}$ at room temperature. 


\section{S5. Carrier lifetime measurements.}

Nanosecond transient absorption was performed using $400 \mathrm{~nm}$ ultrafast pump pulse along with an electronically delayed supercontinuum light source with a sub-nanosecond pulse duration (EOS, Ultrafast Systems). We measured the carrier lifetimes of four perovskite thin films using nanosecond transient absorption spectroscopy at very low pump fluence $\left(<10^{-17} \mathrm{~cm}^{-3}\right.$ in carrier density) and fitted the kinetics using convoluted multi-exponential function. As shown in Figure S5, the carrier lifetimes vary in different samples and they are much longer than spin lifetimes.
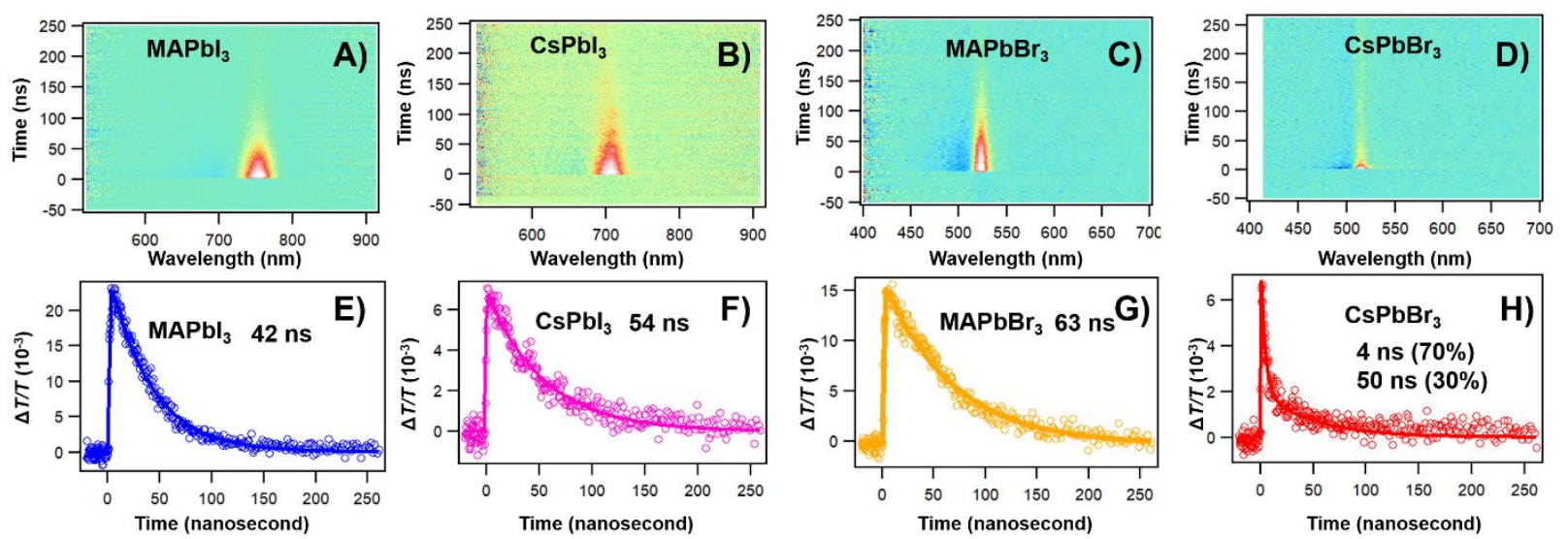

Figure S5. (A-D) Nanosecond TA data map and (E-H) corresponding fits probed at GSB peak of different perovskite films. 
S6. UV-vis absorption spectra and spin dynamics of $\mathrm{MAPbIBr}_{2}$ and $\mathrm{MAPbI}_{2} \mathrm{Br}$.
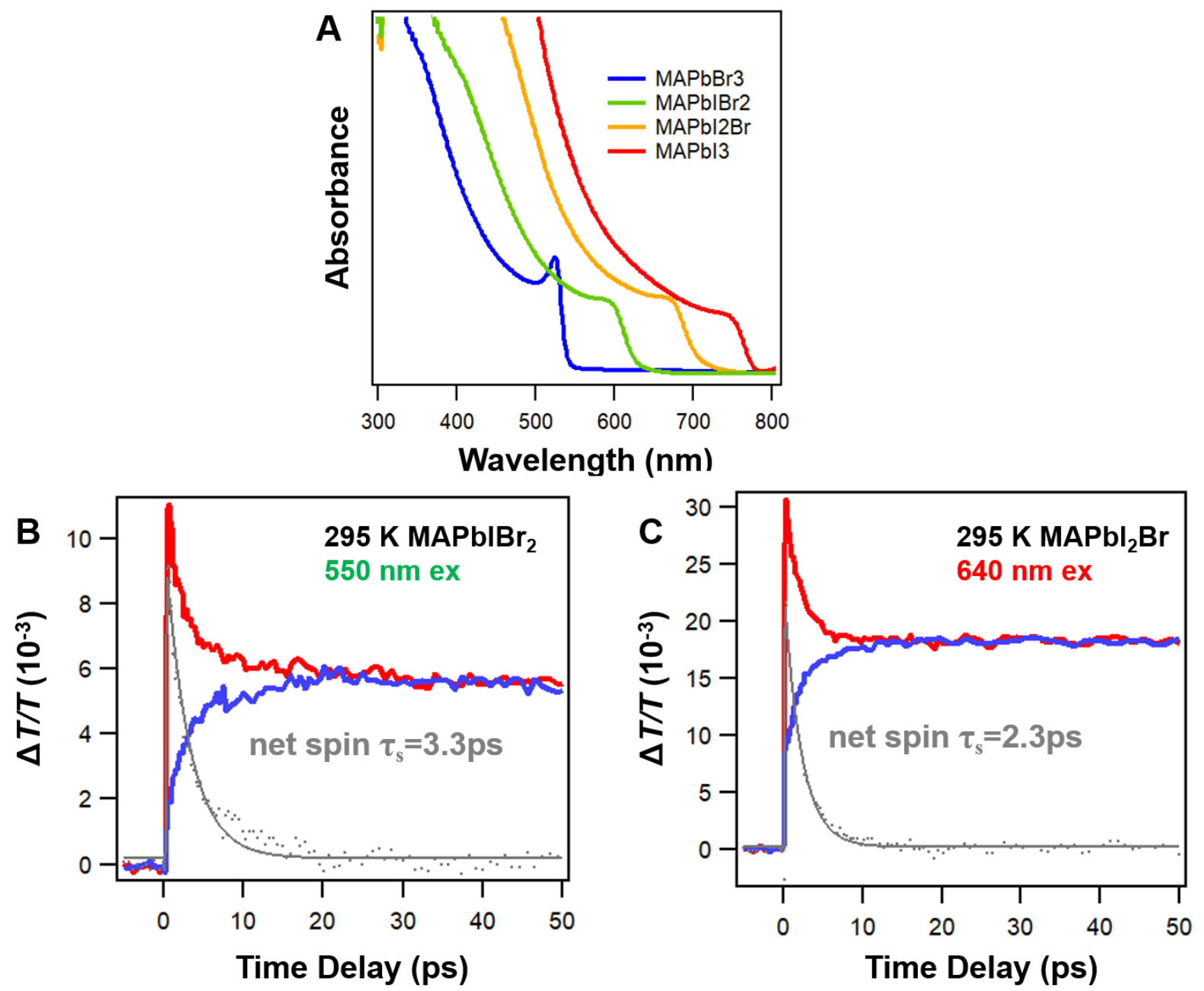

Figure S6. (A) UV-vis absorption spectra of four different films; (B) spin dynamics of $\mathrm{MAPbIBr} 2$ at room temperature; (C) spin dynamics of $\mathrm{MAPbI}_{2} \mathrm{Br}$ at room temperature. 
S7. Spin dynamics of MAPbI 3 at different temperatures pumped at $710 \mathbf{n m}$.
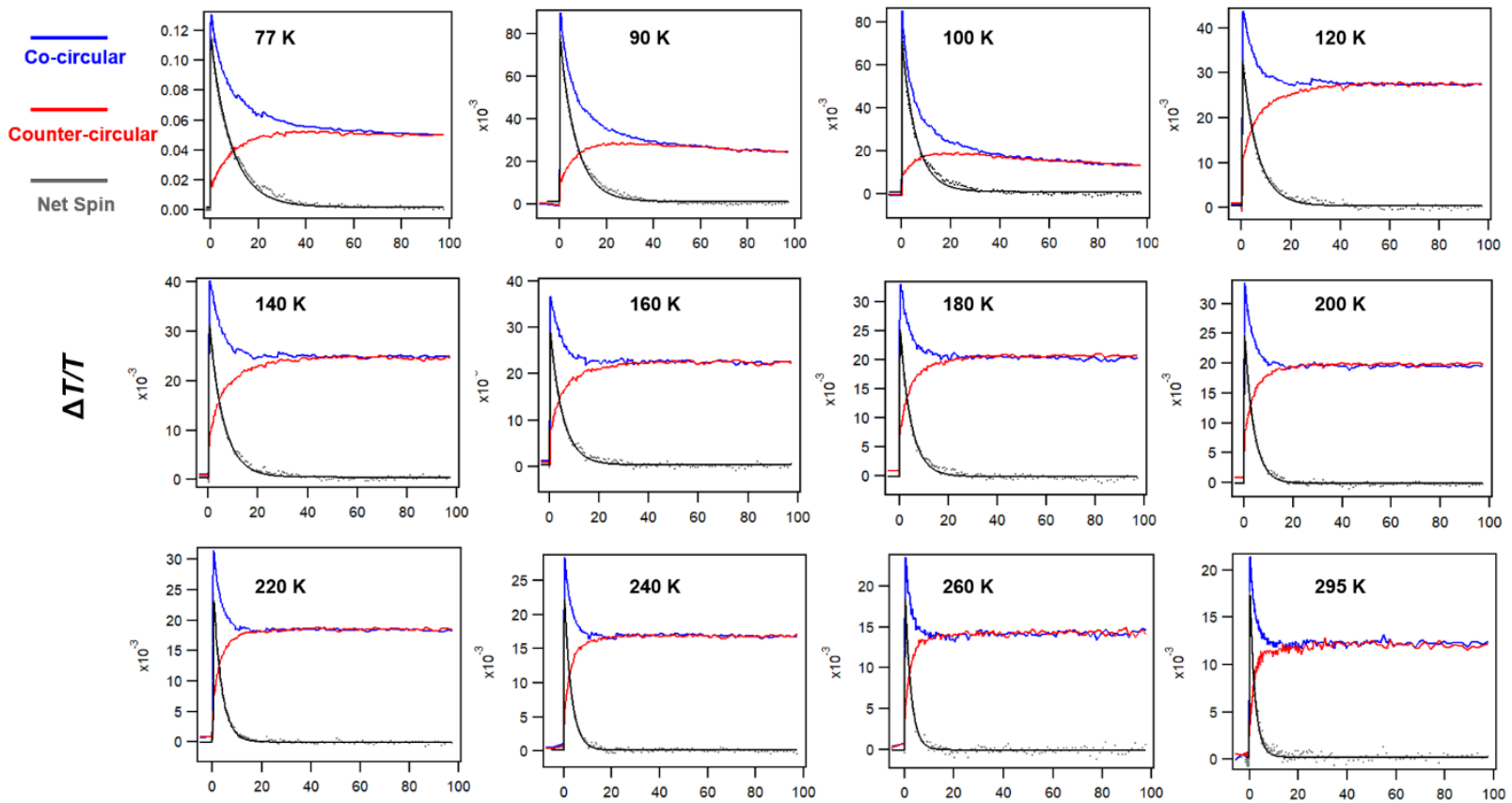

Time Delay (ps)

Figure S7. Transient absorption kinetics of co- and counter-circular polarization as well as net spin dynamics and corresponding fit between 77 and $295 \mathrm{~K}$ for $\mathrm{MAPbI}_{3}$. 
S8. Spin dynamics of $\mathrm{CsPbI}_{3}$ at different temperatures pumped at $710 \mathrm{~nm}$.
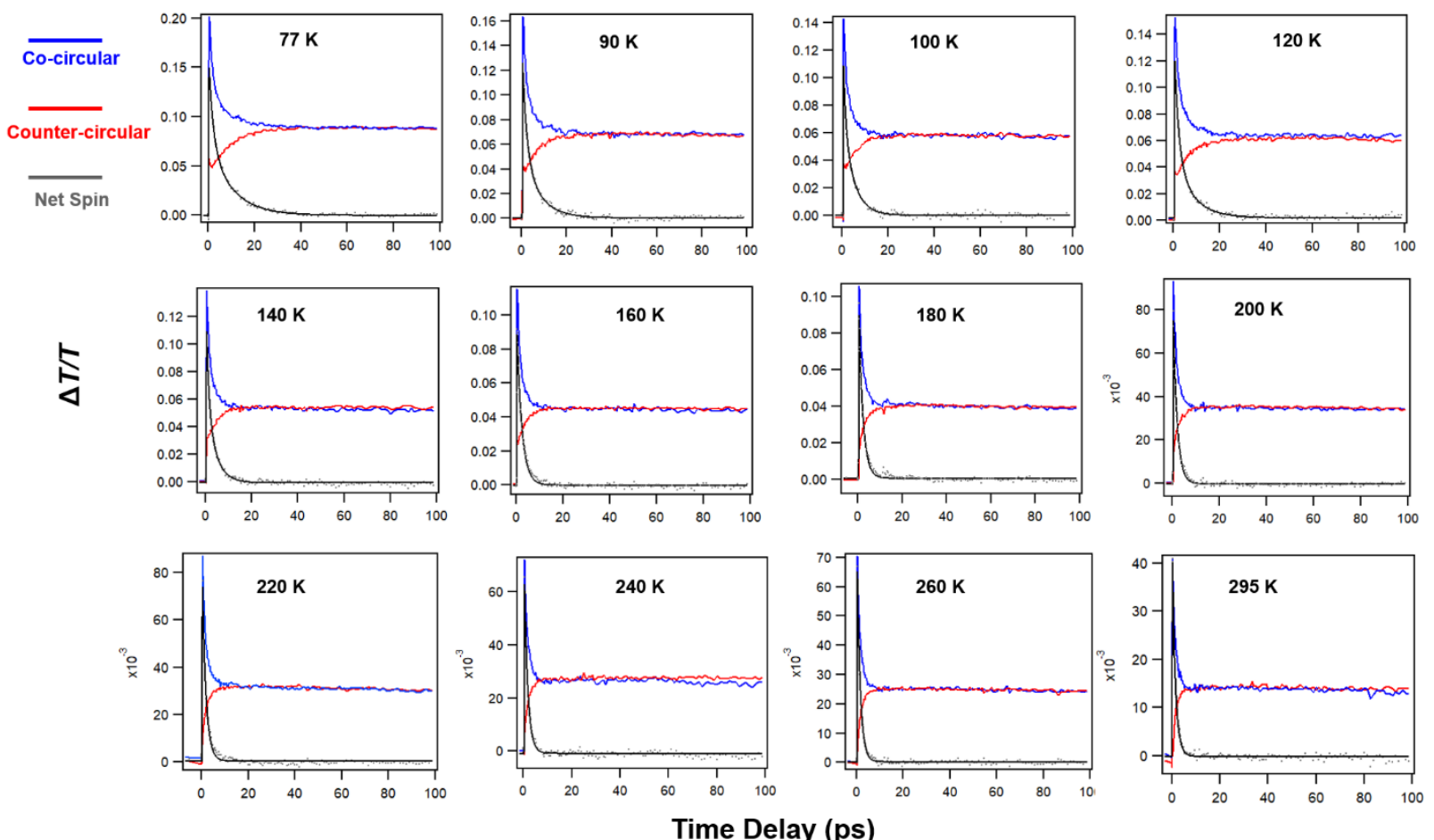

Figure S8. Transient absorption kinetics of co- and counter-circular polarization as well as net spin dynamics and corresponding fit between 77 and $295 \mathrm{~K}$ for $\mathrm{CsPb}_{3}$. 
S9. Spin dynamics of MAPbBr 3 at different temperatures pumped at $490 \mathrm{~nm}$.
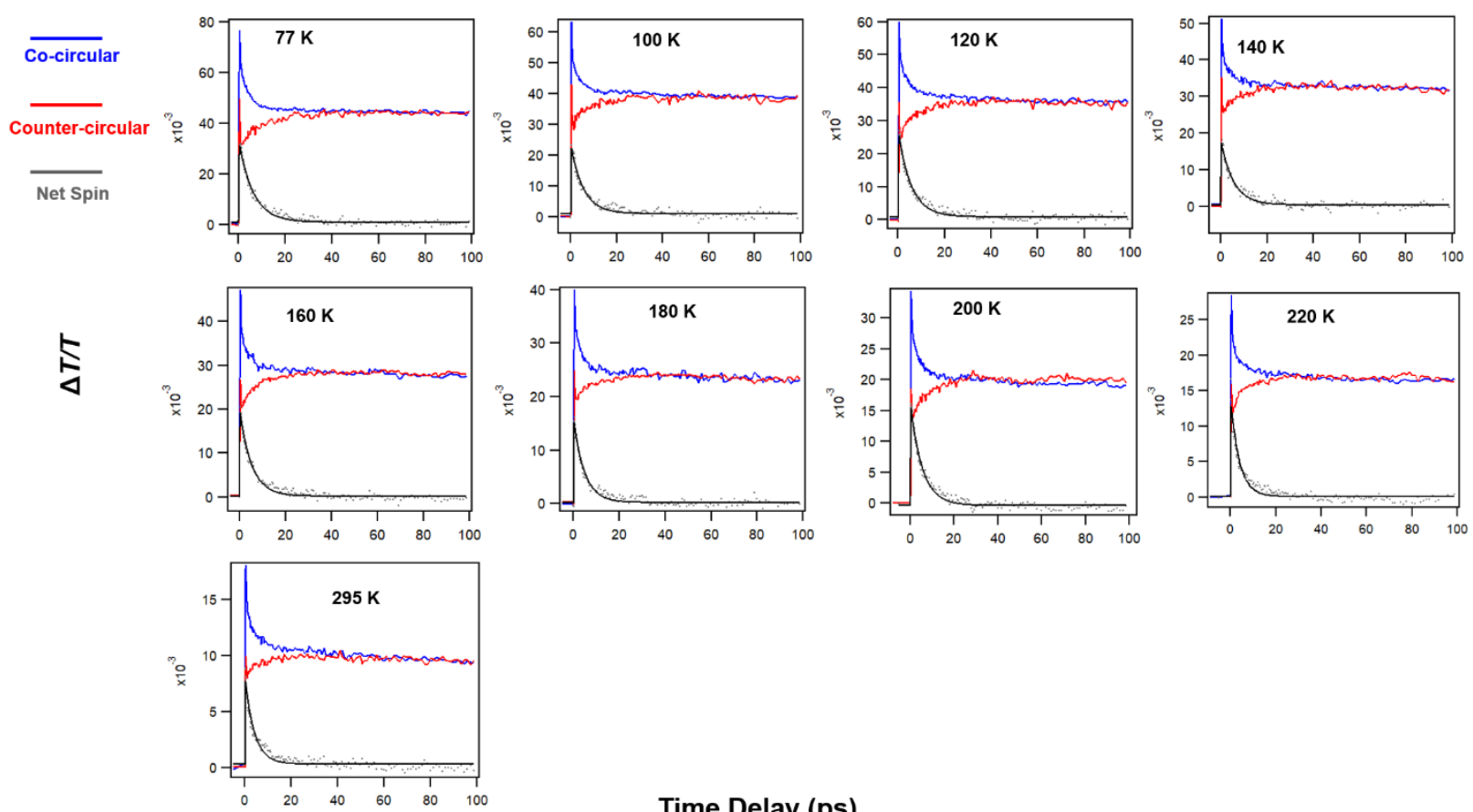

Time Delay (ps)

Figure S9. Transient absorption kinetics of co- and counter-circular polarization as well as net spin dynamics and corresponding fit between 77 and $295 \mathrm{~K}$ for $\mathrm{MAPbBr}_{3}$. 
$\mathrm{S10}$. Spin dynamics of $\mathrm{CsPbBr}_{3}$ at different temperatures pumped at $490 \mathrm{~nm}$.
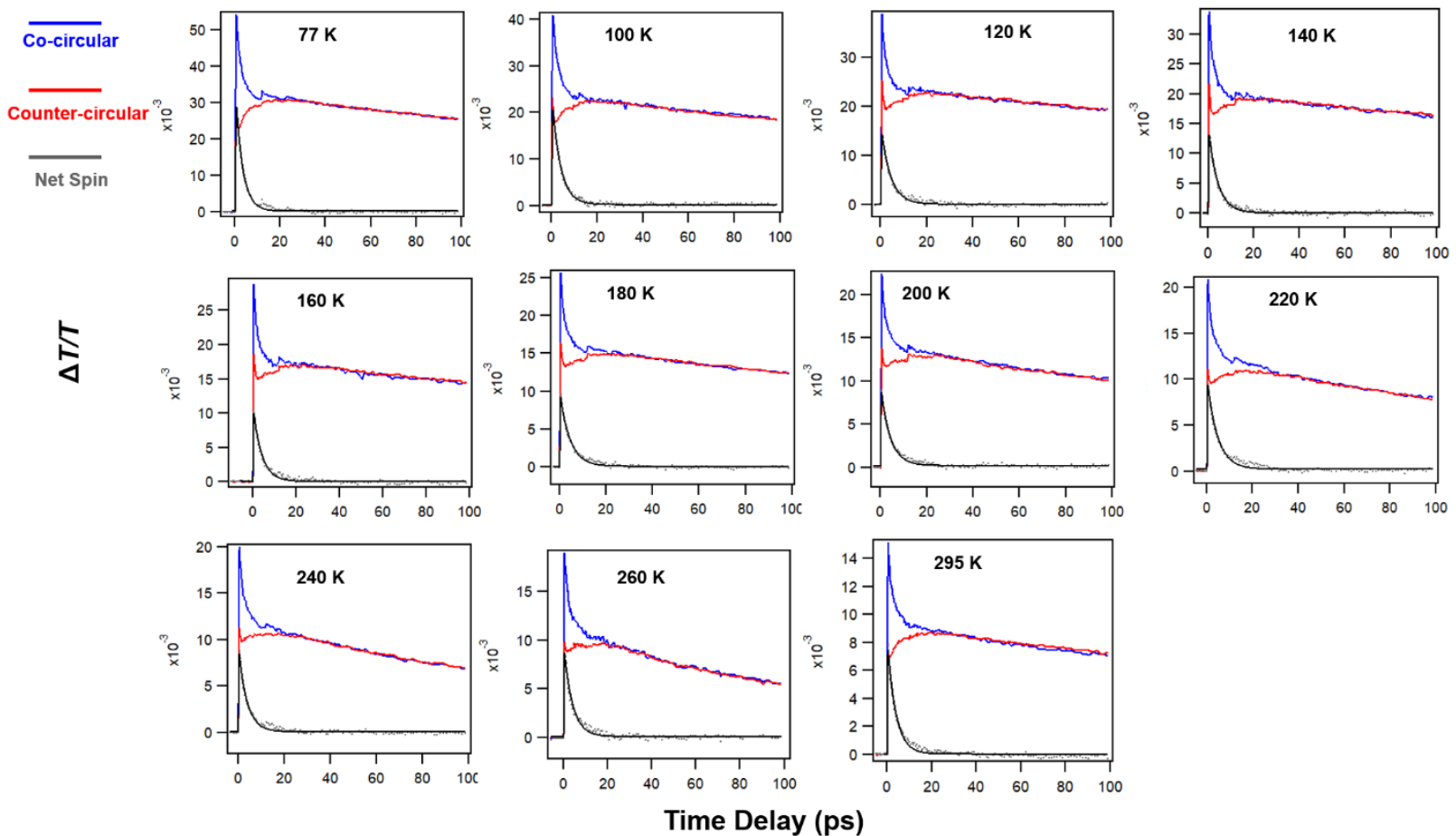

Figure S10. Transient absorption kinetics of co- and counter-circular polarization as well as net spin dynamics and corresponding fit between 77 and $295 \mathrm{~K}$ for $\mathrm{CsPbBr}_{3}$. 
S11. Repeated low temperature measurement on MAPbI3.

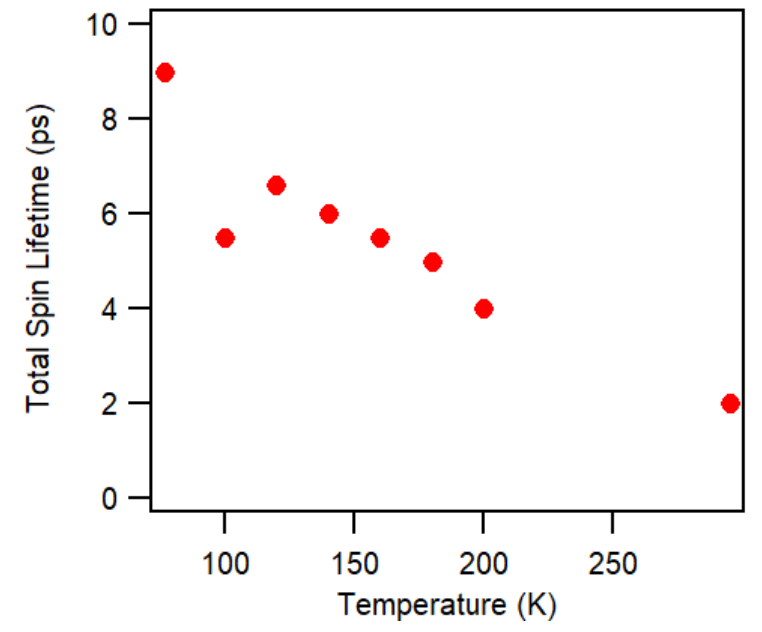

Figure S11. Spin lifetime versus temperature of $\mathrm{MAPbI}_{3}$ in a separate experiment to check the reproducibility. 
S12. Low Temperature Photoluminescence (PL) of MAPbI 3 and MAPbBr3.

The temperature dependent PL linewidth $\Gamma$ can be approximately written as $\Gamma=\Gamma_{0}+\gamma_{a c} T+$ $\gamma_{L O} \mathrm{~N}_{L O}(T)+\gamma_{\text {imp }} e^{-E b / k b T},{ }^{9}$ where $\Gamma_{0}$ is the intrinsic line width; $\gamma_{\text {ac }}$ represents the acoustic phonon coupling term; $\gamma_{\mathrm{LO}}$ is the optical phonon coupling term; $\gamma_{\text {imp }}$ is the impurity term; $N_{L O}(\mathrm{~T})$ is the occupation number of phonons. It is found that the linewidth has a linear relationship with temperature, we thus omit the contribution of optical phonon and impurity and used $\Gamma=\Gamma_{0}+\gamma T$ to fit the linewidth versus temperature. For $\mathrm{MAPbBr}_{3}, \gamma=0.01$ and in $\mathrm{MAPbI}_{3}, \gamma=0.008$.
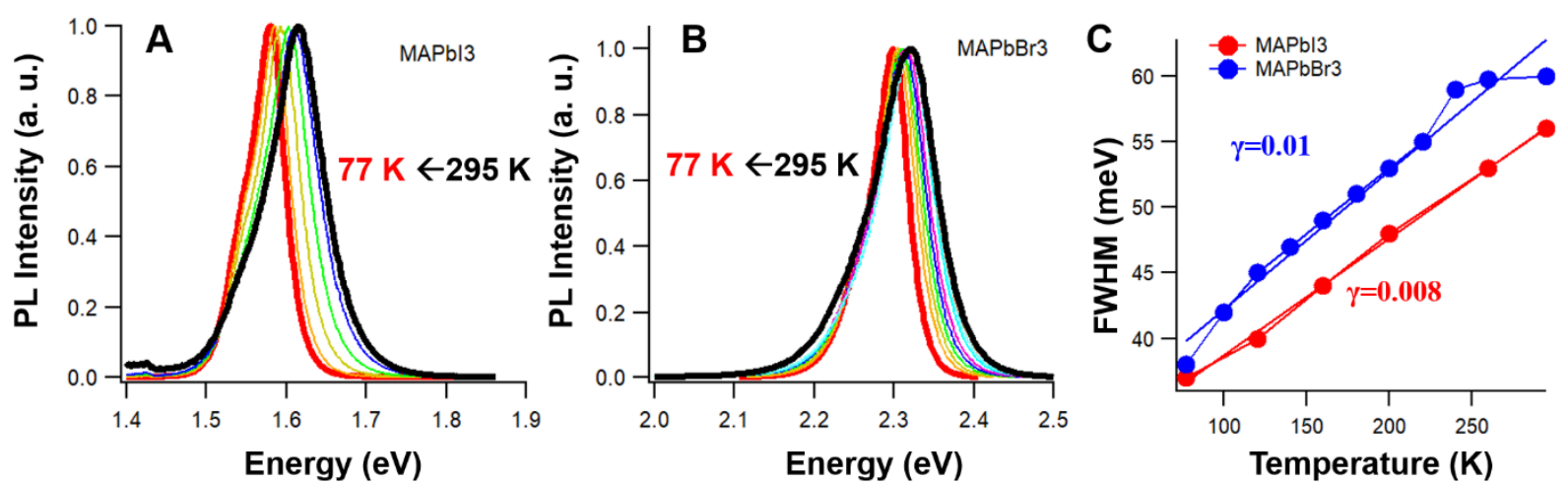

Figure S12. (A) PL spectra of $\mathrm{MAPbI}_{3}$ between 295 and $77 \mathrm{~K}$; (B) PL spectra of $\mathrm{MAPbBr}_{3}$ between 295 and $77 \mathrm{~K}$; (C) PL peak width of both perovskites versus temperature and corresponding fits. It is found that $\mathrm{MAPbBr}_{3}$ exhibits a stronger phonon coupling strength compared to that of $\mathrm{MAPbI}_{3}$. 


\section{References.}

(1) Xiang, S.; Fu, Z.; Li, W.; Wei, Y.; Liu, J.; Liu, H.; Zhu, L.; Zhang, R.; Chen, H., Highly Air-Stable Carbon-Based $\alpha-\mathrm{CsPbI}_{3}$ Perovskite Solar Cells with a Broadened Optical Spectrum. ACS Energy Lett. 2018, 3, 1824-1831.

(2) Huertas-Hernando, D.; Guinea, F.; Brataas, A., Spin-Orbit-Mediated Spin Relaxation in Graphene. Phys. Rev. Lett. 2009, 103, 146801.

(3) Song, L.; Guo, X.; Hu, Y.; Lv, Y.; Lin, J.; Liu, Z.; Fan, Y.; Liu, X., Efficient Inorganic Perovskite Light-Emitting Diodes with Polyethylene Glycol Passivated Ultrathin CsPbBr 3 Films. J. Phys. Chem. Lett. 2017, 8, 4148-4154.

(4) Cho, H.; Wolf, C.; Kim, J. S.; Yun, H. J.; Bae, J. S.; Kim, H.; Heo, J.-M.; Ahn, S.; Lee, T.-W., High-Efficiency Solution-Processed Inorganic Metal Halide Perovskite Light-Emitting Diodes. Adv. Mater. 2017, 29, 1700579.

(5) Chen, X.; Lu, H.; Yang, Y.; Beard, M. C., Excitonic Effects in Methylammonium Lead Halide Perovskites. $J$. Phys. Chem. Lett. 2018, 9, 2595-2603.

(6) Yang, Y.; Yang, M.; Li, Z.; Crisp, R.; Zhu, K.; Beard, M. C., Comparison of Recombination Dynamics in $\mathrm{CH}_{3} \mathrm{NH}_{3} \mathrm{PbBr}_{3}$ and $\mathrm{CH}_{3} \mathrm{NH}_{3} \mathrm{PbI}_{3}$ Perovskite Films: Influence of Exciton Binding Energy. J. Phys. Chem. Lett. 2015, 6, 4688-4692.

(7) Elliott, R. J., Intensity of Optical Absorption by Excitons. Phy. Rev. 1957, 108, 1384-1389.

(8) Giovanni, D.; Ma, H.; Chua, J.; Grätzel, M.; Ramesh, R.; Mhaisalkar, S.; Mathews, N.; Sum, T. C., Highly Spin-Polarized Carrier Dynamics and Ultralarge Photoinduced Magnetization in $\mathrm{CH}_{3} \mathrm{NH}_{3} \mathrm{PbI}_{3}$ Perovskite Thin Films. Nano Lett. 2015, 15, 1553-1558.

(9) Wright, A. D.; Verdi, C.; Milot, R. L.; Eperon, G. E.; Pérez-Osorio, M. A.; Snaith, H. J.; Giustino, F.; Johnston, M. B.; Herz, L. M., Electron-phonon coupling in hybrid lead halide perovskites. Nat. Commun. 2016, 7, 11755. 